\title{
On large Toeplitz band matrices with an uncertain block
}

\author{
A. Böttcher ${ }^{a, 1}$, M. Embree ${ }^{b, 2}$ and V. I. Sokolov ${ }^{a, 3}$ \\ ${ }^{\mathrm{a}}$ Fakultät für Mathematik, TU Chemnitz, 09107 Chemnitz, Germany \\ ${ }^{\mathrm{b}}$ Oxford University Computing Laboratory, Wolfson Building, Parks Road, \\ Oxford OX1 3QD, UK
}

\begin{abstract}
This report investigates the possible spectra of large, finite dimensional Toeplitz band matrices with perturbations (impurities, uncertainties) in the upper-left $m \times m$ block. The main result shows that the asymptotic spectrum of such a matrix is not affected by these perturbations, provided they have sufficiently small norm. This follows from analysis of structured pseudospectra (structured spectral value sets). In contrast, for typical non-Hermitian Toeplitz matrices there exist certain rankone perturbations of arbitrarily small norm that move an eigenvalue away from the asymptotic spectrum in the large-dimensional limit.
\end{abstract}

\section{Introduction}

Let $A$ be a bounded linear operator on $\ell^{2}(\mathbf{N})$ or on $\mathbf{C}^{n}$ with the $\ell^{2}$ norm. We denote the spectrum of $A$ by sp $A$. For a natural number $m$, let $P_{m}$ be the projection on $\ell^{2}(\mathbf{N})$ or $\mathbf{C}^{n}$ that sends a sequence $x=\left\{x_{k}\right\}$ to the sequence defined by $\left(P_{m} x\right)_{k}=x_{k}$ for $1 \leq k \leq m$ and $\left(P_{m} x\right)_{k}=0$ for $k \geq m+1$. We set

$$
\mathrm{sp}_{\varepsilon}^{m} A=\bigcup_{\|K\| \leq \varepsilon} \operatorname{sp}\left(A+P_{m} K P_{m}\right)
$$

Thus, $\mathrm{sp}_{\varepsilon}^{m} A$ measures the extent to which sp $A$ can increase by a perturbation (impurity, uncertainty) of norm at most $\varepsilon$ localized in the upper-left $m \times m$ block of $A$.

1 Email: aboettch@mathematik.tu-chemnitz.de.

2 Email: mark.embree@comlab.ox.ac.uk. Supported by UK Engineering and Physical Sciences Research Council Grant GR/M12414.

3 Email: sokolov@mathematik.tu-chemnitz.de. 
Sets like $\operatorname{sp}_{\varepsilon}^{m} A$ are referred to as structured pseudospectra or structured spectral value sets. These sets have been introduced and applied to problems of linear systems theory by Hinrichsen, Kelb, Pritchard, Gallestey and others (see, e.g., $[9,15,16])$. Consider, for example, the system $\dot{x}=A x+P_{m} v, y=P_{m} x$, in which $A$ is a large or infinite matrix and only the first $m$ components of the input and the output are available. The problem of the existence of a feedback $v=K y$ with $\|K\| \leq \varepsilon$ for which the resulting system operator has $\lambda$ in its spectrum is equivalent to the question of whether $\lambda \in \operatorname{sp}_{\varepsilon}^{m} A$.

Here, we study the case where $A$ is a Toeplitz band matrix. Random perturbations to such matrices (typically on the entire main diagonal, or in a single entry) arise in non-Hermitian quantum mechanics [6-8,11,13], population dynamics [18], and small world networks [21], for instance. A deterministic version arises in the study of initial-boundary value problems, where a finitedifference discretization yields a Toeplitz matrix plus a corner perturbation corresponding to boundary conditions $[1,5,17]$. Pseudospectral analysis was applied to a specific bidiagonal example in [23], while structured pseudospectra were first used for perturbed Toeplitz matrices in our papers [2,3].

In [2], we showed that if $A$ is an infinite non-diagonal Toeplitz band matrix, then there exists an $\varepsilon_{0}=\varepsilon_{0}(A, m)>0$ such that $\operatorname{sp}_{\varepsilon}^{m} A=\operatorname{sp} A$ for all $\varepsilon \in\left(0, \varepsilon_{0}\right)$. This reveals that sufficiently small uncertainties, localized in a fixed finite set of sites, cannot change the spectrum of $A$. Here we prove an analogous result for large finite Toeplitz band matrices. This is particularly interesting because the spectra of typical non-Hermitian finite Toeplitz band matrices are very sensitive to perturbations; the resolvent norm grows exponentially in the matrix dimension, $n$, at points well separated from the asymptotic spectrum [19]. In the large- $n$ limit of these finite matrices, certain rank-one perturbations of arbitrarily small norm move an eigenvalue to any point where the resolvent norm grows without bound in $n$ [22]. The present work demonstrates a class of rank- $m$ perturbations of finite norm that fail to move the eigenvalues anywhere beyond the asymptotic spectrum.

Single-entry perturbations of large finite Toeplitz matrices are the focus of [3]. Let $e_{j} e_{k}^{*}$ be the matrix that is zero everywhere except in the $(j, k)$ entry, which equals 1 . The single-entry analogue of (1) is

$$
\operatorname{sp}_{\varepsilon}^{(j, k)} A=\bigcup_{\omega \in \varepsilon \overline{\mathbf{D}}} \operatorname{sp}\left(A+\omega e_{j} e_{k}^{*}\right)
$$

where $\varepsilon \overline{\mathbf{D}}=\{z \in \mathbf{C}:|z| \leq \varepsilon\}$. In [3], we used the well-known equality

$$
\operatorname{sp}_{\varepsilon}^{(j, k)} A=\operatorname{sp} A \cup\left\{\lambda \in \mathbf{C} \backslash \operatorname{sp} A:\left|\left[(A-\lambda I)^{-1}\right]_{k j}\right| \geq 1 / \varepsilon\right\}
$$


to determine $\operatorname{sp}_{\varepsilon}^{(j, k)} A$ when $A$ is a large Toeplitz band matrix. The analogue of (2) for the structured pseudospectra (1) is the formula

$$
\operatorname{sp}_{\varepsilon}^{m} A=\operatorname{sp} A \cup\left\{\lambda \in \mathbf{C} \backslash \operatorname{sp} A:\left\|P_{m}(A-\lambda I)^{-1} P_{m}\right\| \geq 1 / \varepsilon\right\} .
$$

This formula, established only recently by Gallestey, Hinrichsen, and Pritchard in [9], is the key to our present analysis.

\section{Main result}

For a complex-valued continuous function $a$ on the complex unit circle $\mathbf{T}$, the infinite Toeplitz matrix $T(a)$ and the finite Toeplitz matrices $T_{n}(a)$ are defined by

$$
T(a)=\left(a_{j-\ell}\right)_{j, \ell=1}^{\infty} \quad \text { and } \quad T_{n}(a)=\left(a_{j-\ell}\right)_{j, \ell=1}^{n},
$$

where $a_{k}$ is the $k$ th Fourier coefficient of $a$,

$$
a_{k}=\frac{1}{2 \pi} \int_{0}^{2 \pi} a\left(e^{i \theta}\right) e^{-i k \theta} d \theta, \quad k \in \mathbf{Z} .
$$

Here, we require $a$ to be a trigonometric polynomial, $a \in \mathcal{P}$, which means that at most a finite number of the Fourier coefficients are nonzero, or, equivalently, that $T(a)$ is a banded matrix. The matrix $T(a)$ induces a bounded operator on $\ell^{2}(\mathbf{N})$, and we think of $T_{n}(a)$ as a bounded operator on $\mathbf{C}^{n}$ with the $\ell^{2}$ norm.

The spectrum $\operatorname{sp} A$ of a bounded linear operator is the set of all $\lambda \in \mathbf{C}$ for which $A-\lambda I$ is not invertible. The spectrum of $T(a)$ was identified by Gohberg in 1952 [10]:

$$
\operatorname{sp} T(a)=a(\mathbf{T}) \cup\{\lambda \in \mathbf{C} \backslash a(\mathbf{T}): \operatorname{wind}(a, \lambda) \neq 0\},
$$

where wind $(a, \lambda)$ is the winding number of $a$ (on the counter-clockwise oriented unit circle) about $\lambda$. Each point in $\operatorname{sp} T(a) \backslash a(\mathbf{T})$ is an eigenvalue of finite multiplicity, while a point on $a(\mathbf{T})$ may be an eigenvalue or not.

Suppose that $a \in \mathcal{P}$ and that $T(a)$ is not triangular. Thus we can write

$$
a(t)=\sum_{k=-p}^{q} a_{k} t^{k}, \quad p \geq 1, \quad q \geq 1, \quad a_{-p} a_{q} \neq 0 .
$$


Theorem 2.1 If a is of the form (5), then

$$
\operatorname{sp}_{\varepsilon}^{m} T(a)=\operatorname{sp} T(a) \quad \text { for all } \varepsilon \in\left(0, \varepsilon_{1}\right)
$$

where

$$
\varepsilon_{1}=\max \left(\left|a_{-p}\right|,\left|a_{q}\right|\right)\left(\sum_{j=0}^{m-1}(m-j) p^{2 j}\right)^{-1 / 2}\left(\sum_{j=0}^{m-1}(m-j) q^{2 j}\right)^{-1 / 2} .
$$

A slightly different (and more general) version of this theorem was established in our paper [2]. For the reader's convenience, we give a proof of Theorem 2.1 in Section 4.

The spectra of $T_{n}(a)$ for large $n$ were characterized by Schmidt and Spitzer in 1960 [20]. They showed that the sets $\operatorname{sp} T_{n}(a)$ converge in the Hausdorff metric to some "very thin" set $\Lambda(a)$,

$$
\lim _{n \rightarrow \infty} \operatorname{sp} T_{n}(a)=\Lambda(a)
$$

and they gave the following description of $\Lambda(a)$. It is clear that $\Lambda(a)=\left\{a_{0}\right\}$ if $T(a)$ is triangular. So suppose that $T(a)$ is not triangular and $a$ is given by (5). For $\varrho>0$, define $a_{\varrho} \in \mathcal{P}$ by $a_{\varrho}(t)=\sum_{k=-p}^{q} a_{k} \varrho^{k} t^{k}$. In [20], it was shown that

$$
\Lambda(a)=\bigcap_{\varrho>0} \operatorname{sp} T\left(a_{\varrho}\right)
$$

If $T(a)$ is triangular, then, obviously,

$$
\operatorname{sp}_{\varepsilon}^{m} T_{n}(a) \supset \operatorname{sp}_{\varepsilon}^{1} T_{n}(a)=a_{0}+\varepsilon \overline{\mathbf{D}} .
$$

This implies that the limit of $\operatorname{sp}_{\varepsilon}^{m} T_{n}(a)$ as $n \rightarrow \infty$ is strictly larger than $\Lambda(a)=\left\{a_{0}\right\}$ for every $\varepsilon>0$. As the following theorem shows, this cannot happen for non-triangular Toeplitz band matrices.

Theorem 2.2 If a is of the form (5), then there exists an $\varepsilon_{2}=\varepsilon_{2}(a, m)>0$ such that

$$
\lim _{n \rightarrow \infty} \operatorname{sp}_{\varepsilon}^{m} T_{n}(a)=\Lambda(a) \quad \text { for all } \varepsilon \in\left(0, \varepsilon_{2}\right),
$$

the limit being taken in the Hausdorff metric. 
Theorems 2.1 and 2.2 show that $\operatorname{sp}_{\varepsilon}^{m} T(a)$ and $\lim \mathrm{sp}_{\varepsilon}^{m} T_{n}(a)$ stabilize at constant sets before $\varepsilon$ reaches zero and that, moreover, these two constant sets are in general different. Thus, except for some trivial cases, we always have

$$
\lim _{n \rightarrow \infty} \operatorname{sp}_{\varepsilon}^{m} T_{n}(a) \varsubsetneqq \operatorname{sp}_{\varepsilon}^{m} T(a)
$$

for sufficiently small $\varepsilon$, implying that the passage from the "finite volume case" to the "infinite volume case" is discontinuous.

\section{An example}

We provide a concrete demonstration of these results for the symbol $a(t)=$ $t+t^{-2}$. In this case, the set $\lim _{n \rightarrow \infty} \operatorname{sp} T_{n}(a)=\Lambda(a)$ consists of three line segments joined at the origin,

$$
\Lambda(a)=\left\{r e^{i \theta}: 0 \leq r \leq 3 \cdot 2^{-2 / 3}, \theta \in\left\{0, \frac{2 \pi}{3}, \frac{4 \pi}{3}\right\}\right\}
$$

see [20, Section 7]. Figure 1 illustrates this set, together with $\operatorname{sp} T(a)$.

Now consider perturbations to the upper-left $3 \times 3$ block of $T_{n}(a)$, i.e., $m=3$. We abbreviate $P_{3} K P_{3}$ to $E$. Figure 2 shows the eigenvalues of $T_{50}(a)+E$ for 1000 values of $E$, where $E$ has complex normally distributed entries scaled so that $\|E\|=\varepsilon$ for $\varepsilon=\frac{1}{2}, \varepsilon=\frac{3}{4}$, and $\varepsilon=1$. These sets suggest $\operatorname{sp}_{\varepsilon}^{3} T_{50}(a)$. Note that $\operatorname{sp}_{\varepsilon}^{3} T_{50}(a)$ closely resembles $\Lambda(a)$ for $\varepsilon=\frac{1}{2}$, while $\operatorname{sp}_{\varepsilon}^{3} T_{50}(a)$ deviates from $\Lambda(a)$ as $\varepsilon$ increases. Figure 3 offers more convincing evidence, illustrating the maximum distance that some eigenvalue of $T_{n}(a)+E$ varies from $\Lambda(a)$,

$$
\max _{\lambda \in \operatorname{sp}\left(T_{n}(a)+E\right)} \operatorname{dist}(\lambda, \Lambda(a)):=\max _{\lambda \in \operatorname{sp}\left(T_{n}(a)+E\right)} \min _{z \in \Lambda(a)}|\lambda-z|,
$$

as a function of $\varepsilon=\|E\|$ for $n=25$ and $n=100$. (For each fixed $\varepsilon$, we take the maximum over 1000 randomly-generated perturbations E.) For values of $\varepsilon$ roughly less than $\frac{4}{10}$, the perturbed eigenvalues don't differ much from the asymptotic spectrum, while for larger values of $\varepsilon$ this difference appears to grow linearly with $\varepsilon$. As the matrix dimension increases, the difference between $\operatorname{sp}\left(T_{n}(a)+E\right)$ and $\Lambda(a)$ reduces for small $\varepsilon$. (Note that $T_{3}(a)$ is a normal matrix, so that $\operatorname{sp}\left(T_{3}(a)+E\right)$ can vary from $\Lambda(a)$ by $\varepsilon$ for all $\varepsilon \geq 0$.) The computations presented here were executed with MATLAB. 

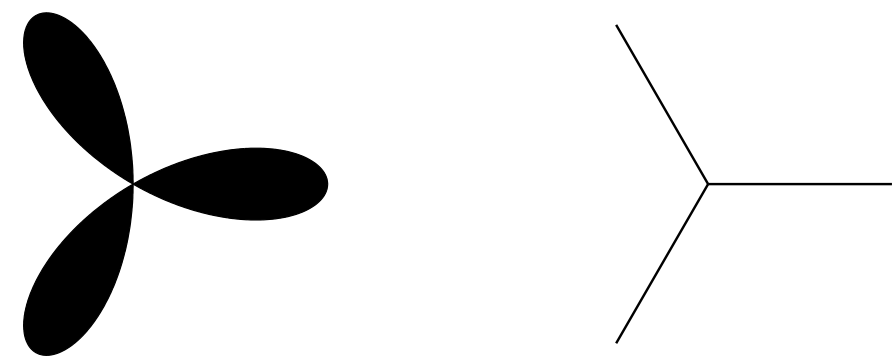

Fig. 1. The sets $\operatorname{sp} T(a)$ (left) and $\lim _{n \rightarrow \infty} \operatorname{sp} T_{n}(a)=\Lambda(a)$ (right) for $a(t)=t+t^{-2}$.
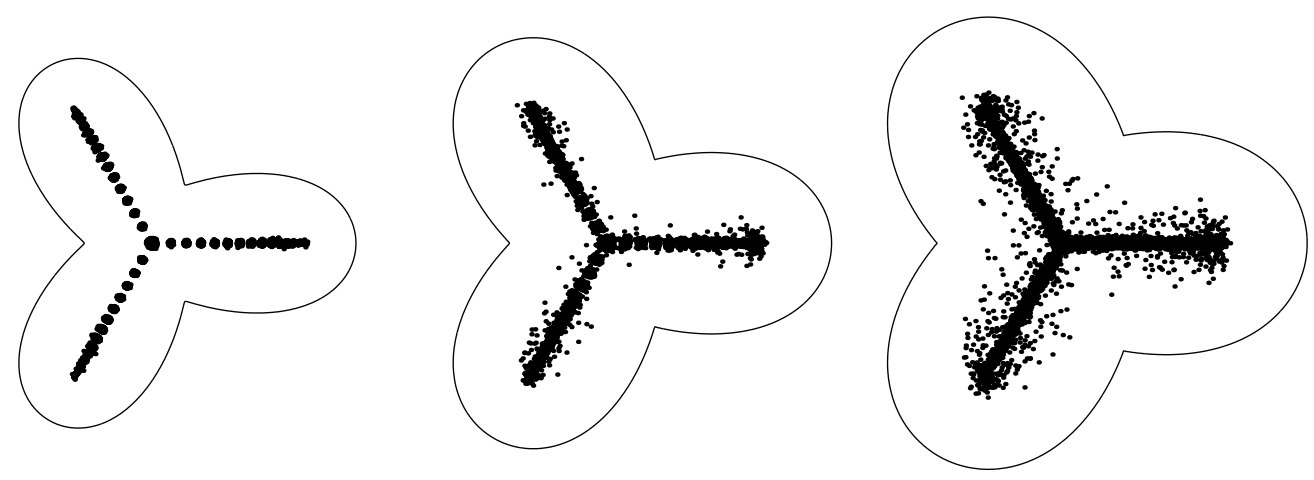

Fig. 2. Superimposed eigenvalues of $T_{50}(a)+E$ (with $a(t)=t+t^{-2}$ ) for 1000 complex random perturbations $E$ of norm $\frac{1}{2}, \frac{3}{4}$, and 1 . The solid curves on each plot reveal the boundary of $\operatorname{sp}_{\varepsilon}^{50} T_{50}(a)$, computed using the software [24].

\section{Proofs}

Proof of Theorem 2.1. Pick $\lambda \in \mathbf{C} \backslash \operatorname{sp} T(a)$. We have

$$
a(t)-\lambda=t^{-p} a_{q}\left(t-z_{1}(\lambda)\right) \ldots\left(t-z_{p+q}(\lambda)\right)
$$

and since $a-\lambda$ has no zeros on $\mathbf{T}$, we can write

$$
a(t)-\lambda=t^{-p} a_{q}\left(t-\delta_{1}\right) \ldots\left(t-\delta_{r}\right)\left(t-\mu_{1}\right) \ldots\left(t-\mu_{s}\right)
$$

where $\left|\delta_{j}\right|=\left|\delta_{j}(\lambda)\right|<1$ and $\left|\mu_{j}\right|=\left|\mu_{j}(\lambda)\right|>1$. As wind $(a, \lambda)=-p+r$ and wind $(a, \lambda)=0$ due to $(4)$, we see that necessarily $p=r$ and hence $q=s$. Thus, $a(t)-\lambda=a_{q} \varphi_{-}(t) \varphi_{+}(t)$ with

$$
\varphi_{-}(t):=\left(1-\frac{\delta_{1}}{t}\right) \ldots\left(1-\frac{\delta_{p}}{t}\right), \quad \varphi_{+}(t)=\left(t-\mu_{1}\right) \ldots\left(t-\mu_{q}\right) .
$$




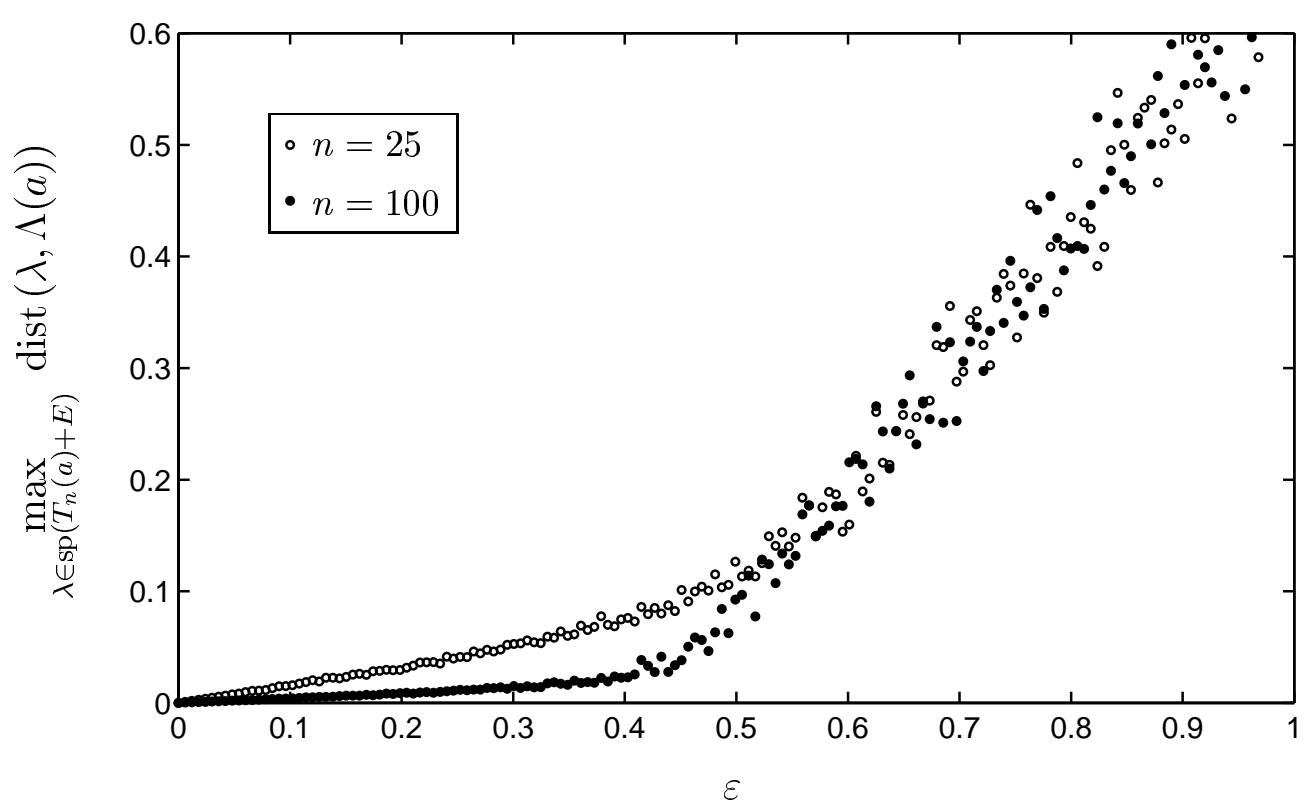

Fig. 3. Deviation of $\operatorname{sp}\left(T_{n}(a)+E\right)$ from $\Lambda(a)$ for the symbol $a(t)=t+t^{-2}$. Each data point represents the maximum distance over 1000 randomly generated perturbations $E$ of norm $\varepsilon$.

Standard computations with Toeplitz matrices (see, e.g., [4]) now give

$$
\begin{aligned}
& T(a-\lambda)=a_{q} T\left(\varphi_{-} \varphi_{+}\right)=a_{q} T\left(\varphi_{-}\right) T\left(\varphi_{+}\right), \\
& T^{-1}(a-\lambda)=a_{q}^{-1} T\left(\varphi_{+}^{-1}\right) T\left(\varphi_{-}^{-1}\right), \\
& P_{m} T^{-1}(a-\lambda) P_{m}=a_{q}^{-1} P_{m} T\left(\varphi_{+}^{-1}\right) T\left(\varphi_{-}^{-1}\right) P_{m}=a_{q}^{-1} T_{m}\left(\varphi_{+}^{-1}\right) T_{m}\left(\varphi_{-}^{-1}\right) .
\end{aligned}
$$

We have

$$
\varphi_{-}^{-1}(t)=\left(1+\frac{\delta_{1}}{t}+\frac{\delta_{1}^{2}}{t^{2}}+\ldots\right) \ldots\left(1+\frac{\delta_{p}}{t}+\frac{\delta_{p}^{2}}{t^{2}}+\ldots\right)=: \sum_{n=0}^{\infty} b_{n} t^{-n}
$$

with

$$
\left|b_{n}\right|=\left|\sum_{\alpha_{1}+\ldots+\alpha_{p}=n} \delta_{1}^{\alpha_{1}} \ldots \delta_{p}^{\alpha_{p}}\right| \leq\left(\left|\delta_{1}\right|+\ldots+\left|\delta_{p}\right|\right)^{n}<p^{n}
$$

and because

$$
T_{m}\left(\varphi_{-}^{-1}\right)=\left(\begin{array}{cccc}
b_{0} & b_{1} & \ldots & b_{m-1} \\
& b_{0} & \ldots & b_{m-2} \\
& & \ddots & \vdots \\
& & & b_{0}
\end{array}\right)
$$


it follows that

$$
\begin{aligned}
\left\|T_{m}\left(\varphi_{-}^{-1}\right)\right\|^{2} & \leq m\left|b_{0}\right|^{2}+(m-1)\left|b_{1}\right|^{2}+\ldots+\left|b_{m-1}\right|^{2} \\
& \leq m+(m-1) p^{2}+\ldots+p^{2 m-2}
\end{aligned}
$$

Analogously,

$$
\left\|T_{m}\left(\varphi_{+}^{-1}\right)\right\|^{2} \leq m+(m-1) q^{2}+\ldots+q^{2 m-2} .
$$

In summary,

$$
\left\|P_{m} T^{-1}(a-\lambda) P_{m}\right\|^{2} \leq \frac{1}{\left|a_{q}\right|^{2}}\left(\sum_{j=0}^{m-1}(m-j) p^{2 j}\right)\left(\sum_{j=0}^{m-1}(m-j) q^{2 j}\right) .
$$

Denote the right-hand side of (10) by $M /\left|a_{q}\right|^{2}$. From (3) we now infer that $\operatorname{sp}_{\varepsilon}^{m} T(a)$ is the union of $\operatorname{sp} T(a)$ and the set

$$
\left\{\lambda \notin \operatorname{sp} T(a):\left\|P_{m} T^{-1}(a-\lambda) P_{m}\right\|^{2} \geq \frac{1}{\varepsilon^{2}}\right\} \subset\left\{\lambda \notin \operatorname{sp} T(a): \frac{M}{\left|a_{q}\right|^{2}} \geq \frac{1}{\varepsilon^{2}}\right\} .
$$

Clearly, the last set is empty whenever $M /\left|a_{q}\right|^{2}<1 / \varepsilon^{2}$, which gives the assertion with $\varepsilon_{1}=\left|a_{q}\right| / \sqrt{M}$. Considering the transpose of $T(a)$, we get the assertion with $\varepsilon_{1}=\left|a_{-p}\right| / \sqrt{M}$. This implies Theorem 2.1 with $\varepsilon_{1}$ given by formula (6).

We now turn to the proof of Theorem 2.2.

Lemma 4.1 There exists a constant $\delta=\delta(a)>1$ such that

$$
\Lambda(a)=\bigcap_{\varrho \in[1 / \delta, \delta]} \operatorname{sp} T\left(a_{\varrho}\right) .
$$

A proof of this lemma is in [3].

For a sequence $\left\{M_{n}\right\}_{n=1}^{\infty}$ of nonempty compact sets $M_{n} \subset \mathbf{C}$, we consider the two limiting sets

$$
\begin{aligned}
\liminf _{n \rightarrow \infty} M_{n}:=\{\lambda \in \mathbf{C}: & \lambda \text { is the limit of some } \\
& \text { sequence } \left.\left\{\lambda_{n}\right\}_{n=1}^{\infty} \text { with } \lambda_{n} \in M_{n}\right\}, \\
\limsup _{n \rightarrow \infty} M_{n}:=\{\lambda \in \mathbf{C}: & \lambda \text { is a partial limit of some } \\
& \text { sequence } \left.\left\{\lambda_{n}\right\}_{n=1}^{\infty} \text { with } \lambda_{n} \in M_{n}\right\} .
\end{aligned}
$$


It is well known that the two equalities

$$
\liminf _{n \rightarrow \infty} M_{n}=\limsup _{n \rightarrow \infty} M_{n}=M
$$

are equivalent to the convergence of $M_{n}$ to $M$ in the Hausdorff metric (see, e.g., [12, Sections 3.1.1 and 3.1.2] or [14, Section 28]).

Lemma 4.2 For every $\varepsilon>0$,

$$
\limsup _{n \rightarrow \infty} \operatorname{sp}_{\varepsilon}^{m} T_{n}(a) \subset \operatorname{sp}_{\varepsilon}^{m} T(a)
$$

Proof. Pick $\lambda \in \mathbf{C} \backslash \operatorname{sp}_{\varepsilon}^{m} T(a)$. Then $\lambda \notin \operatorname{sp} T(a)$ and, by (3),

$$
\left\|P_{m} T^{-1}(a-\lambda) P_{m}\right\|<1 / \varepsilon .
$$

It follows that there is an open neighborhood $U \subset \mathbf{C}$ of $\lambda$ such that if $\mu \in U$, then $\mu \notin \operatorname{sp} T(a)$ and

$$
\left\|P_{m} T^{-1}(a-\mu) P_{m}\right\|<1 / \varepsilon .
$$

A well-known result on the finite section method for Toeplitz operators (see, e.g., [4, p. 42]) says that $T_{n}^{-1}(a-\mu)$ converges strongly (i.e., pointwise) to $T^{-1}(a-\mu)$. Consequently,

$$
\left\|P_{m} T_{n}^{-1}(a-\mu) P_{m}-P_{m} T^{-1}(a-\mu) P_{m}\right\| \rightarrow 0 \text { as } n \rightarrow \infty,
$$

and it is straightforward to check that the convergence in (11) is uniform with respect to $\mu$ in compact subsets of $U$. Thus, there exist an open neighborhood $V \subset U$ of $\lambda$ and a natural number $n_{0}$ such that

$$
\left\|P_{m} T_{n}^{-1}(a-\mu) P_{m}\right\|<1 / \varepsilon \text { for all } \mu \in V \text { and all } n \geq n_{0} .
$$

This, in conjunction with (3), implies that $V \cap \operatorname{sp}_{\varepsilon}^{m} T(a)=\emptyset$ for all $n \geq n_{0}$, whence $\lambda \notin \limsup _{n \rightarrow \infty} \operatorname{sp}_{\varepsilon}^{m} T(a)$.

Lemma 4.3 Let $\delta$ be the constant given by Lemma 4.1. If $\varrho \in[1 / \delta, \delta]$ and $\varepsilon>0$, then

$$
\limsup _{n \rightarrow \infty} \operatorname{sp}_{\varepsilon}^{m} T_{n}(a) \subset \operatorname{sp}_{\beta}^{m} T\left(a_{\varrho}\right),
$$

where $\beta=\varepsilon \max \left(\varrho^{m+1}, \varrho^{-m-1}\right)$. 
Proof. We have $T_{n}(a-\lambda)=D_{n}^{-1}(\varrho) T_{n}\left(a_{\varrho}-\lambda\right) D_{n}(\varrho)$, where $D_{n}(\varrho)$ is the diagonal matrix $\operatorname{diag}\left(\varrho, \varrho^{2}, \ldots, \varrho^{n}\right)$. It results that

$$
P_{m} T_{n}^{-1}(a-\lambda) P_{m}=D_{m}^{-1}(\varrho) P_{m} T_{n}^{-1}\left(a_{\varrho}-\lambda\right) P_{m} D_{m}(\varrho),
$$

whence

$$
\left\|P_{m} T_{n}^{-1}(a-\lambda) P_{m}\right\| \leq \kappa_{m}(\varrho)\left\|P_{m} T_{n}^{-1}\left(a_{\varrho}-\lambda\right) P_{m}\right\|,
$$

where $\kappa_{m}(\varrho):=\left\|D_{m}^{-1}(\varrho)\right\|\left\|D_{m}(\varrho)\right\|$. Since also $\operatorname{sp} T_{n}(a)=\operatorname{sp} T_{n}\left(a_{\varrho}\right)$, we conclude from (3) that

$$
\begin{aligned}
\operatorname{sp}_{\varepsilon}^{m} T_{n}(a) & =\operatorname{sp} T_{n}(a) \cup\left\{\lambda \notin \operatorname{sp} T_{n}(a):\left\|P_{m} T_{n}^{-1}(a-\lambda) P_{m}\right\| \geq 1 / \varepsilon\right\} \\
& =\operatorname{sp} T_{n}\left(a_{\varrho}\right) \cup\left\{\lambda \notin \operatorname{sp} T_{n}\left(a_{\varrho}\right):\left\|P_{m} T_{n}^{-1}(a-\lambda) P_{m}\right\| \geq 1 / \varepsilon\right\} \\
& \subset \operatorname{sp} T_{n}\left(a_{\varrho}\right) \cup\left\{\lambda \notin \operatorname{sp} T_{n}\left(a_{\varrho}\right): \kappa_{m}(\varrho)\left\|P_{m} T_{n}^{-1}\left(a_{\varrho}-\lambda\right) P_{m}\right\| \geq 1 / \varepsilon\right\} \\
& =\operatorname{sp}_{\varepsilon \kappa_{m}(\varrho)}^{m} T_{n}\left(a_{\varrho}\right),
\end{aligned}
$$

and now Lemma 4.2 yields the inclusion

$$
\limsup _{n \rightarrow \infty} \operatorname{sp}_{\varepsilon}^{m} T_{n}(a) \subset \operatorname{sp}_{\varepsilon \kappa_{m}(\varrho)}^{m} T\left(a_{\varrho}\right) .
$$

Because $\kappa_{m}(\varrho)=\varrho^{m-1}$ if $\varrho \geq 1$ and $\kappa_{m}(\varrho)=\varrho^{-m+1}$ if $\varrho<1$, we arrive at the assertion.

Proof of Theorem 2.2. Denote the right-hand side of (6) by

$$
C(p, q, m) \max \left(\left|a_{-p}\right|,\left|a_{q}\right|\right)
$$

let $\delta$ be the constant from Lemma 4.1, and put

$$
\varepsilon_{2}:=C(p, q, m)\left|a_{q}\right| / \delta^{q+m-1} .
$$

We claim that Theorem 2.2 is true with this choice of $\varepsilon_{2}$.

Let $\varepsilon \in\left(0, \varepsilon_{2}\right)$. Lemma 4.2 shows that if $\varrho \in[1 / \delta, \delta]$, then (12) holds with

$$
\begin{aligned}
\beta & =\varepsilon \max \left(\varrho^{m-1}, \varrho^{-m+1}\right) \\
& <C(p, q, m)\left|a_{q}\right| \max \left(\varrho^{m-1}, \varrho^{-m+1}\right) / \delta^{q+m-1} \\
& \left.\leq C(p, q, m)\left|a_{q}\right| \varrho^{q} \delta^{q} \max \left(\varrho^{m-1}, \varrho^{-m+1}\right) / \delta^{q+m-1} \quad \text { (since } 1 \leq \varrho \delta\right) \\
& =C(p, q, m)\left|a_{q}\right| \varrho^{q} \max \left(\varrho^{m-1}, \varrho^{-m+1}\right) / \delta^{m-1} \\
& \leq C(p, q, m)\left|a_{q}\right| \varrho^{q} .
\end{aligned}
$$


Applying Theorem 2.1 to $T\left(a_{\varrho}\right)$ and taking into account that $\left|a_{q}\right|$ does not exceed $\max \left(\left|a_{-p}\right|,\left|a_{q}\right|\right)$, we see that

$$
\operatorname{sp}_{\beta}^{m} T\left(a_{\varrho}\right)=\operatorname{sp} T\left(a_{\varrho}\right) \quad \text { for } \beta<C(p, q, m)\left|a_{q}\right| \varrho^{q} .
$$

Thus, (12) and (13) give $\lim \operatorname{supsp}_{\varepsilon}^{m} T_{n}(a) \subset \operatorname{sp} T\left(a_{\varrho}\right)$ for every $\varrho \in[1 / \delta, \delta]$. By Lemma 4.1, this implies that

$$
\limsup _{n \rightarrow \infty} \operatorname{sp}_{\varepsilon}^{m} T_{n}(a) \subset \Lambda(a)
$$

To establish inclusion in the other direction, note that (7) implies

$$
\Lambda(a) \subset \liminf _{n \rightarrow \infty} \operatorname{sp} T_{n}(a) \subset \liminf _{n \rightarrow \infty} \operatorname{sp}_{\varepsilon}^{m} T_{n}(a)
$$

thus completing the proof of Theorem 2.2.

\section{References}

[1] R. M. Beam and R. F. Warming, The asymptotic spectra of banded Toeplitz and quasi-Toeplitz matrices, SIAM J. Sci. Comput. 14 (1993) 971-1006.

[2] A. Böttcher, M. Embree, and V. I. Sokolov, Infinite Toeplitz and Laurent matrices with localized impurities, Linear Algebra Appl., to appear.

[3] A. Böttcher, M. Embree, and V. I. Sokolov, The spectra of large Toeplitz band matrices with a randomly perturbed entry, in preparation.

[4] A. Böttcher and B. Silbermann, Introduction to Large Truncated Toeplitz Matrices (Universitext, Springer-Verlag, New York, 1998).

[5] B. Cahlon, D. M. Kulkarni, and P. Shi, Stepwise stability for the heat equation with a nonlocal constraint, SIAM J. Numer. Anal. 32 (1995) 571-593.

[6] E. B. Davies, Spectral properties of random non-self-adjoint matrices and operators, Proc. Roy. Soc. London Ser. A 457 (2001) 191-206.

[7] J. Feinberg and A. Zee, Non-Hermitian localization and delocalization, Phys. Rev. E 59 (1999) 6433-6443.

[8] J. Feinberg and A. Zee, Spectral curves of non-hermitian hamiltonians, Nuc. Phys. B 522 (1999) 599-623.

[9] E. Gallestey, D. Hinrichsen, and A. J. Pritchard, Spectral value sets of closed linear operators, Proc. Roy. Soc. London Ser. A 456 (2000) 1397-1418. 
[10] I. Gohberg, On an application of the theory of normed rings to singular integral equations, Uspekhi Matem. Nauk SSSR 112 (1952) 149-156 [Russian].

[11] I. Ya. Goldsheid and B. A. Khoruzhenko, Distribution of eigenvalues in nonHermitian Anderson models, Phys. Rev. Lett. 80 (1998) 2897-2900.

[12] R. Hagen, S. Roch, and B. Silbermann, $C^{*}$-Algebras and Numerical Analysis (Dekker, New York, 2001).

[13] N. Hatano and D. R. Nelson, Vortex pinning and non-Hermitian quantum mechanics, Phys. Rev. B 77 (1996) 8651-8673.

[14] F. Hausdorff, Set Theory (Chelsea, New York, 1957).

[15] D. Hinrichsen and B. Kelb, Spectral value sets: a graphical tool for robustness analysis, Systems Control Lett. 21 (1993) 127-136.

[16] D. Hinrichsen and A. J. Pritchard, Real and complex stability radii: a survey, in: D. Hinrichsen and B. Mårtensson, eds., Control of Uncertain Systems (Prog. Systems Control Theory, Vol. 6, Birkhäuser Verlag, Basel, 1990) 119-162.

[17] D. Kulkarni, D. Schmidt, and S.-K. Tsui, Eigenvalues of tridiagonal pseudoToeplitz matrices, Linear Algebra Appl. 297 (1999) 63-80.

[18] D. R. Nelson and N. M. Shnerb, Non-Hermitian localization and population biology, Phys. Rev. E 58 (1998) 1383-1403.

[19] L. Reichel and L. N. Trefethen, Eigenvalues and pseudo-eigenvalues of Toeplitz matrices, Linear Algebra Appl. 162-164 (1992) 153-185.

[20] P. Schmidt and F. Spitzer, The Toeplitz matrices of an arbitrary Laurent polynomial, Math. Scand. 8 (1960) 15-38.

[21] G. Strang, X. Liu, S. Ott, and L. He, Localized eigenvectors from localized matrix modifications, in preparation; see also G. Strang, "From the SIAM President", SIAM News, April 2000, May 2000.

[22] L. N. Trefethen, Spectra and pseudospectra: the behavior of non-normal matrices and operators, in: M. Ainsworth, J. Levesley, and M. Marletta, eds., The Graduate Student's Guide to Numerical Analysis '98, (Springer-Verlag, Berlin, 1999) 217-250.

[23] L. N. Trefethen, M. Contedini, and M. Embree, Spectra, pseudospectra, and localization for random bidiagonal matrices, Comm. Pure Appl. Math. 54 (2001) 594-623.

[24] T. G. Wright, MATLAB Pseudospectra GUI, 2001. Available online at: http://web.comlab.ox.ac.uk/oucl/work/tom.wright/psgui/index.html. 